\title{
ESTUDO DO COMPORTAMENTO VIBRACIONAL DE MOTOR A COMBUSTÃO INTERNA SUBMETIDO A DESBALANCEAMENTO EM DIFERENTES POSIÇÕES POR MEIO DE TRANSFORMADA DE WAVELET
}

\author{
Elen Kássia de Assis ${ }^{(1)}$ (elen.kassia@engenharia.ufjf.br), Marcel Henrique Lopes ${ }^{(1)}$ \\ (marcel.henrike@ @otmail.com), Jorge Nei Brito ${ }^{(2)}$ (brito@ufsj.edu.br), José Antônio da Silva ${ }^{(3)}$ \\ (jant@ufsj.edu.br) \\ (1) Univ. Federal de São João Del Rei (UFSJ) - Grupo de Estudos e Pesquisas do Laboratório de Sistemas Dinâmicos (Gep_LASID) \\ (2) Universidade Federal de São João Del Rei (UFSJ) - Departamento de Engenharia Mecânica (DEMEC) \\ ${ }^{(3)}$ Universidade Federal de São João Del Rei (UFSJ) - Departamento de Ciências Térmicas e dos Fluidos (DCTEF)
}

RESUMO: A análise de vibração é um dos métodos de manutenção preditiva mais utilizados para identificação de falhas. É amplamente utilizada para motores elétricos, entretanto sua aplicação a motores a combustão interna é mais complexa, devido à dificuldade em desenvolver um padrão. Este estudo tem por finalidade caracterizar o padrão vibracional do motor Robin EH17-2D da Subaru com base em diferentes posições de desbalanceamento. Os dados vibracionais foram coletados com o equipamento IMX-S 16 , da $S K F$, com o auxílio de acelerômetros piezelétricos. Os espectros vibracionais foram gerados a partir do software @ptitude Analyst, também da SKF. O software MatLab foi utilizado para implementação de um algoritmo de tratamento de sinal por meio da Transformada de Wavelet, que permitiu a caracterização da energia vibracional de cada banda de frequência. Para a exposição gráfica no trabalho, foram selecionadas as posições em que os valores de energia e as alterações no padrão vibracional se mostraram mais relevantes. Conhecendo-se o modo operante do motor em condições propensas à falha, é possivel, por meio de acompanhamento preditivo, identificar, quando este apresentar frequências anômalas, que há uma falha iminente.

PALAVRAS-CHAVE: Manutenção preditiva. Vibração. Motor a combustão interna. Desbalanceamento. Transformada de Wavelet.

\section{INTRODUÇÃO}

A análise de vibração tem o intuito de diagnosticar um problema mecânico e sua origem, a partir de um quadro de sintomas determinado pelas características da vibração. A análise por assinatura de vibração é um dos métodos mais aplicados na identificação de falhas. A sua premissa básica é de que existem certas características espectrais que, em termos gerais, identificam determinados tipos de defeitos (Rezende, 1991).

De acordo com Golwine et al. (2004), a análise da assinatura de vibração no bloco do motor e a análise da variação de torque e potência do motor estão entre as principais abordagens sugeridas para se medir a qualidade da combustão entre os diferentes cilindros do motor. Também de acordo com Golwine et al. (2004), informações sobre a qualidade da combustão em um motor de combustão interna proporcionam uma forte ferramenta de diagnóstico no que diz respeito à operação do motor e sua saúde. 
Segundo Geng et al. (2003), os impactos transitórios gerados pelos sistemas mecânicos internos do motor tendem a ser amplamente distribuídos periodicamente na velocidade de operação do motor quando eles causam respostas ressonantes de alta frequência das peças mecânicas impactantes. A resposta ressonante de alta frequência possui composições de frequência constante e se transforma sucessivamente em uma parte da vibração do motor.

A vibração do processo de combustão é gerada devido à reação de explosão da mistura ar combustível sob altas temperaturas e pressões no interior da câmara de combustão.

A análise de vibração tem sido amplamente utilizada na detecção de falhas em motores elétricos, onde se compara a assinatura de vibração do motor em condições ideias de funcionamento com a assinatura de vibração do motor em que se induziu falha. Através desta comparação é possível determinar as frequências determinísticas de falha, utilizadas na manutenção preditiva. Este tipo de análise, entretanto, ainda não é comum para motores à combustão interna devido ao alto grau de dificuldade em se conseguir repetitividade nos testes e, portanto, em se obter a Assinatura de Vibração (Assis et al., 2018).

O desbalanceamento em máquinas e equipamentos pode ocasionar um gasto energético excessivo e desencadear outros modos de falha, portanto é importante conhecer os alarmes deste tipo de falha a fim de identificar quando ele está ocorrendo em motores a combustão interna.

Este fenômeno é caracterizado pela existência de desequilíbrios de massa em relação aos eixos de rotação. Tais desequilíbrios são originados por assimetrias, tolerâncias e desvios de forma, além das imperfeições da matéria-prima e da montagem (Silva, 2012).

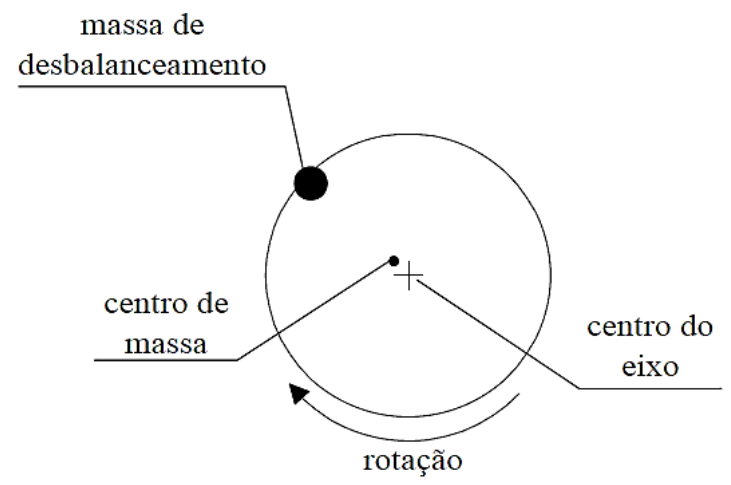

Figura 1. Desequilíbrio de massa. Fonte: Brito (2002).

Baccarini (2005) explica que o desbalanceamento gera uma força que é estacionária em relação ao rotor. O sinal no tempo é senoidal e cada ciclo tem a mesma amplitude e depende da quantidade de desbalanceamento. Assim, a vibração predominante ocorre na frequência de rotação do rotor e terá amplitudes maiores nas direções radiais (horizontal e vertical).

Os motores a combustão interna costumeiramente possuem dois eixos árvores: o virabrequim e o eixo comando de válvulas. Em um motor de quatro tempos e um cilindro, a relação entre as frequências de rotação do virabrequim e de combustão é de 2:1, ou seja, a cada duas voltas do virabrequim ocorre uma combustão. Na análise de vibração deste tipo de motor, estas frequências possuem grandes amplitudes e, por isso, influenciam muito o nível de vibração observado, sendo importantes no estudo desejado. Por serem sinais altamente transientes e limitados a uma banda de 
frequência específica, a análise por decomposição de wavelet apresenta inúmeras vantagens, pois possibilita eliminar da análise as frequências que influenciam o nível de vibração, mas que não são relacionadas com a combustão. As altas frequências geradas por impactos fluidodinâmicos, por exemplo, não dependem da condição de operação, mas incrementam o nível de vibração, podendo levar a erros de diagnóstico neste estudo (Lacerda, 2014).

De acordo com Porfírio (2011), as Transformadas Wavelets Discretas são muito utilizadas para análise de sinais digitais e compressão de dados, e representam a decomposição de uma função no domínio do tempo em um conjunto de coeficientes que descrevem os seus componentes de frequência em determinados instantes de tempo. Com o auxílio da Transformada de Wavelet é possível filtrar os sinais e captar informações mais precisas sobre determinados pontos de oscilação.

Uma wavelet é uma pequena onda de natureza oscilatória e energia finita. Diferentemente de outras técnicas de análise de sinais no domínio da frequência, como a Transformada de Fourier (FT) e a Transformada de Fourier de Curta Duração (STFT), que decompõem o sinal como se ele fosse resultado do somatório de várias ondas senoidais de diferentes frequências, a Transformada de Wavelet (TW) interpreta um sinal como versões deslocadas e escalonadas de uma wavelet original (ou wavelet mãe), Lacerda et al. (2011).

A análise de wavelet é capaz de revelar aspectos dos sinais que outras técnicas de análise não detectam, como descontinuidades, batidas, transições bruscas, início e fim de eventos e eventos de curta duração. Outra diferença entre estas duas técnicas de análise é o tamanho das janelas utilizadas no processo e o efeito delas sobre a resolução do sinal transformado. A análise de wavelet permite o uso de janelas com longos intervalos de tempo onde necessita-se de informações mais precisas em baixas frequências e intervalos menores onde procuramos informações em altas frequências (Lacerda, 2014).

A Árvore de Wavelet referente à decomposição que é feita na parte experimental deste trabalho, onde o sinal é separado em $2^{\wedge} \mathrm{j}$ bandas de frequência, é apresentada na Figura 2 . Cada vetor é chamado de nó ou packet.

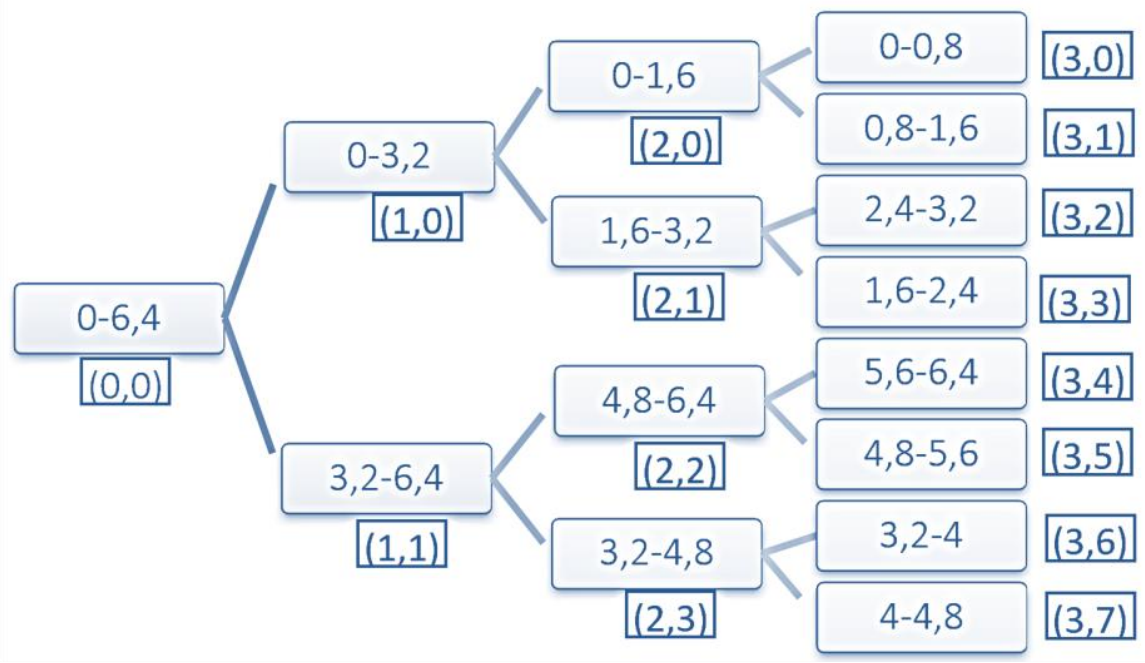

Figura 2. Decomposição por Transformada de Wavelet Packet de nível três (kHz). Fonte: Autores. 
Os sinais coletados nas diferentes condições foram decompostos através da WPT pela Família Daubechies, chamadas de dbN. Estas são wavelets ortonormais de suporte compacto não simétricas, com tamanho $\mathbf{2} \mathbf{N}$ e suporte em $[-\mathbf{N}+\mathbf{1}, \mathbf{N}]$, onde $\mathbf{N}$ é o número de momentos nulos da onda resultante.

Liu e Huang (2005, apud Lacerda, 2016) propõem o método de determinação dos autovalores de energia de cada nó para a detecção de alterações nos coeficientes da wavelet e define a Equação 1 para este fim, onde $\mathbf{E} m$ é o autovalor de energia em cada banda de frequência do nível $\mathbf{j}, \mathbf{C}_{\mathbf{j}, \mathbf{n}}$ é a amplitude em cada ponto discreto do coeficiente da Wavelet Packet do sinal na frequência correspondente e $\mathbf{m}=\mathbf{0}, \mathbf{1}, \mathbf{2} \ldots \mathbf{2}^{\mathbf{j}} \mathbf{\mathbf { 1 }} \mathbf{1}$. Assim tem-se um autovetor de energia dado pela Equação 2, onde $E=\left(\sum_{m=0}^{2^{j}-1}\left|E_{m}\right|^{2}\right)^{1 / 2}$.

$$
\begin{gathered}
E_{m}=\sum_{n=0}^{2^{j}-1}\left|c_{j, n}(t)\right|^{2} \\
T=\left[\frac{E_{0}}{E}, \frac{E_{1}}{E}, \ldots, \frac{E_{2^{j}-1}}{E}\right]
\end{gathered}
$$

\section{MATERIAIS E MÉTODOS}

\subsection{Motor de Estudo}

Os testes foram realizados no Laboratório de Energia do Departamento de Ciências Térmicas e dos Fluidos - DCTEF da Universidade Federal de São João Del Rei - UFSJ. Foi utilizada uma bancada dinamométrica Tecquipment - TQ, Figura 3, composta de um freio hidráulico modelo TD200, equipada com placa de aquisição de dados e instrumentação que permite com o uso dos softwares VDAS e ECA100, análise de torque, potência, pressão atmosférica e rotação do motor.

O motor de estudo é o motor a gasolina da marca Subaru modelo Robin EH17-2D, monocilindro, a gasolina, naturalmente aspirado e refrigerado a ar, mostrado na Figura 3.

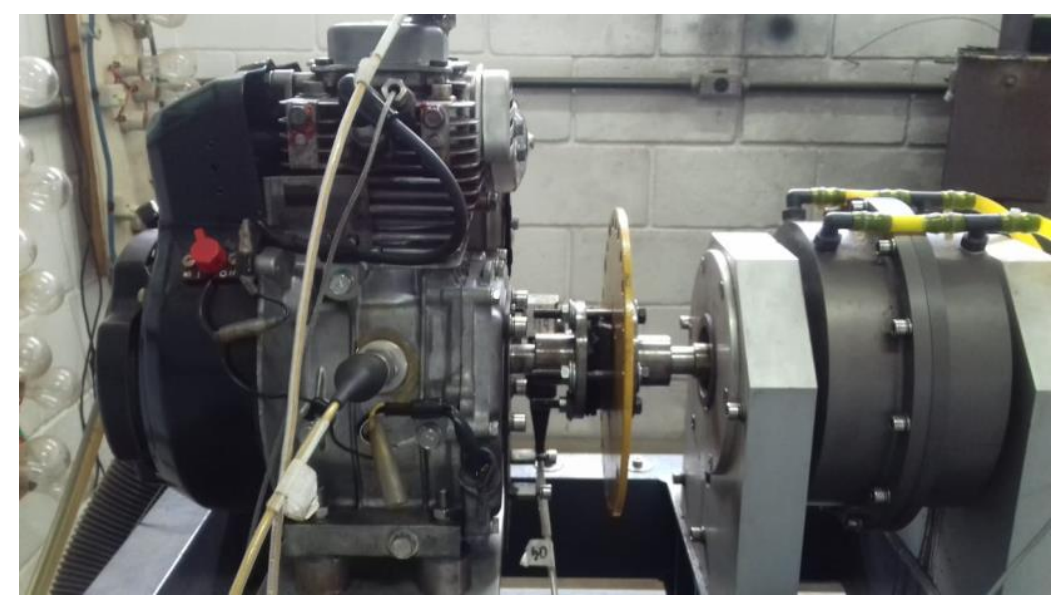

Figura 3. Bancada dinamométrica. Fonte: Autores. 

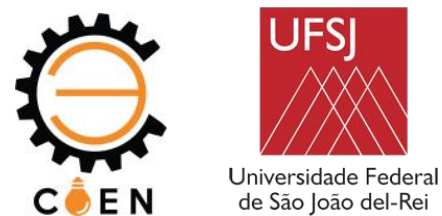

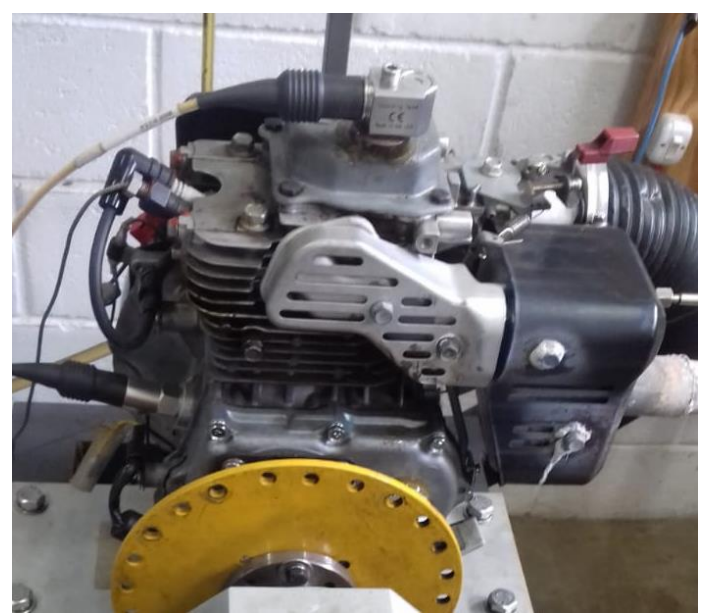

Figura 4. Direção axial do motor de estudo. Fonte: Autores.

\section{$2.2 \quad I M x-16 ~ S$}

O IMX-S 16 é uma tecnologia SKF capaz de coletar dados através de diferentes sensores (acelerômetros, alicate amperímetro, bobina de fluxo magnético dentre outros) e enviar em tempo real para um banco de dados que pode ser acessado remotamente. $\mathrm{O}$ software de armazenamento permite que rotas de monitoramento sejam criadas e que os dados sejam analisados através de suas várias ferramentas disponíveis, o que facilita o diagnóstico dos problemas analisados. Para cada ponto de coleta de vibração podem ser gerados espectros de deslocamento, velocidade, aceleração e envelope simultaneamente.

Os sinais foram coletados por acelerômetros SKF CMSS2200 nas posições Vertical e Axial, e um acelerômetro SKF CMSS2100 na posição Horizontal.

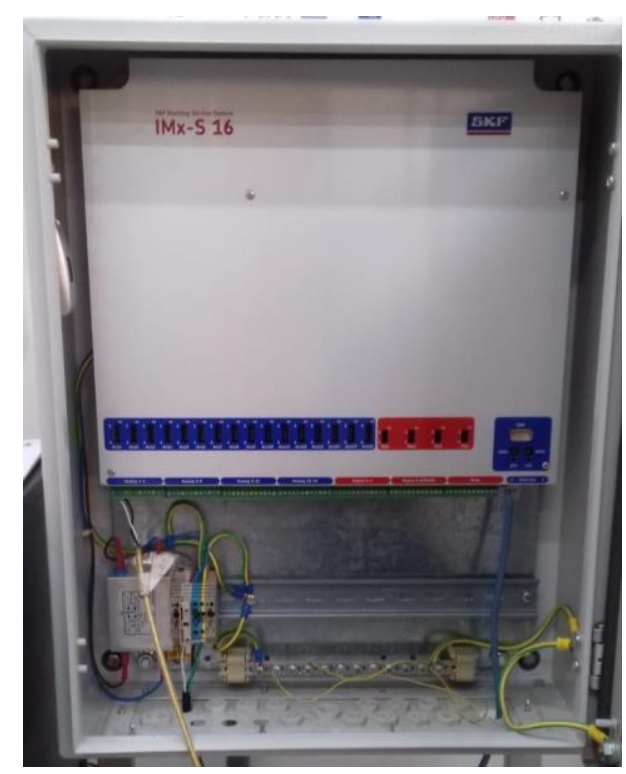

Figura 5. Sistema de aquisição de dados - IMx-S 16. Fonte: Autores. 


\subsection{Software SKF @ ptitude Analyst}

Elaborou-se um banco de dados contendo as faixas de frequência, unidades de medida, tipo de coleta, velocidade de trabalho e componentes relevantes para caracterização da frequência de vibração. A velocidade de trabalho foi fixada em 3000 RPM, a frequência limite adotada foi $5000 \mathrm{~Hz}$, o corte de frequência se deu em $6,25 \mathrm{~Hz}$ e o sinal representativo resulta de quatro médias.

Os sinais coletados foram aceleração, velocidade e envelope, sendo as duas primeiras por meio de detecção RMS e a última por pico. A frequência de amostragem resultante desse banco de dados foi $12800 \mathrm{~Hz}$. Conectado via ethernet ao IMx-S 16, o computador que abriga o software @ ptitude Analyst armazenou os dados provenientes dos acelerômetros, identificando a data e horário no qual o sinal foi coletado. O software permite a plotagem de espectros e gráficos para melhor visualização das faixas vibracionais da máquina em estudo.

As coletas foram feitas para o motor em condição padrão de funcionamento, e para desbalanceamento em $0^{\circ}, 180^{\circ}$ e $270^{\circ}$ (sentido horário) em relação ao pistão em Ponto Morto Superior (PMS).

\subsection{Decomposição dos Sinais}

Os sinais de vibração coletados foram exportados para o software MATLAB® e implementados em um algoritmo de processamento do sinal através da WPT. Para a decomposição foi utilizada a Família Daubechies. Dessa família, foi utilizada a Subfamília db4. Aos sinais decompostos foi aplicado o critério de autovalores para detecção de distúrbios nas bandas de frequência de interesse. O $3^{\circ}$ nível foi o escolhido para análise. A Árvore de Wavelet, Figura 2, representa a decomposição feita neste experimento e a disposição das bandas de frequência dos nós terminais.

\section{RESULTADOS E CONCLUSÕES}

Os testes foram realizados com a rotação fixa em 3000 RPM, e o valor de torque aplicado foi $2 \mathrm{Nm}$, simulando aplicação de carga. O disco foi desbalanceado com uma massa de 13,09g em três posições diferentes do disco, sendo elas, $0^{\circ}, 180^{\circ}$ e $270^{\circ}$ (sentido horário) em relação ao pistão em Ponto Morto Superior (PMS).

Conforme os espectros apresentados, é possível observar a variação da vibração de acordo com a posição do desbalanceamento.

Considerando valores de velocidade, as posições horizontal e axial foram as que apresentaram resultados relevantes para este estudo. Entretanto, para valores de aceleração, as posições vertical e axial se mostraram mais relevantes. 
Seja seu próprio

Diferencial

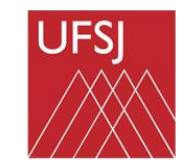

Universidade Federal

C $\mathbf{E N}$

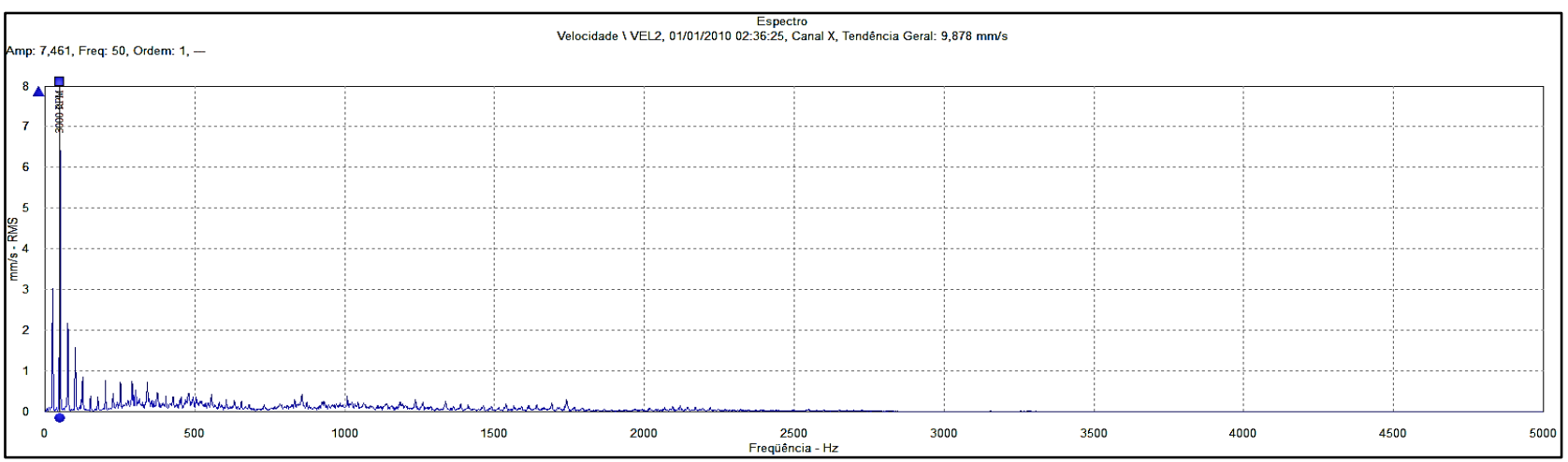

Figura 6. Velocidade horizontal no modo ideal. Fonte: Autores.

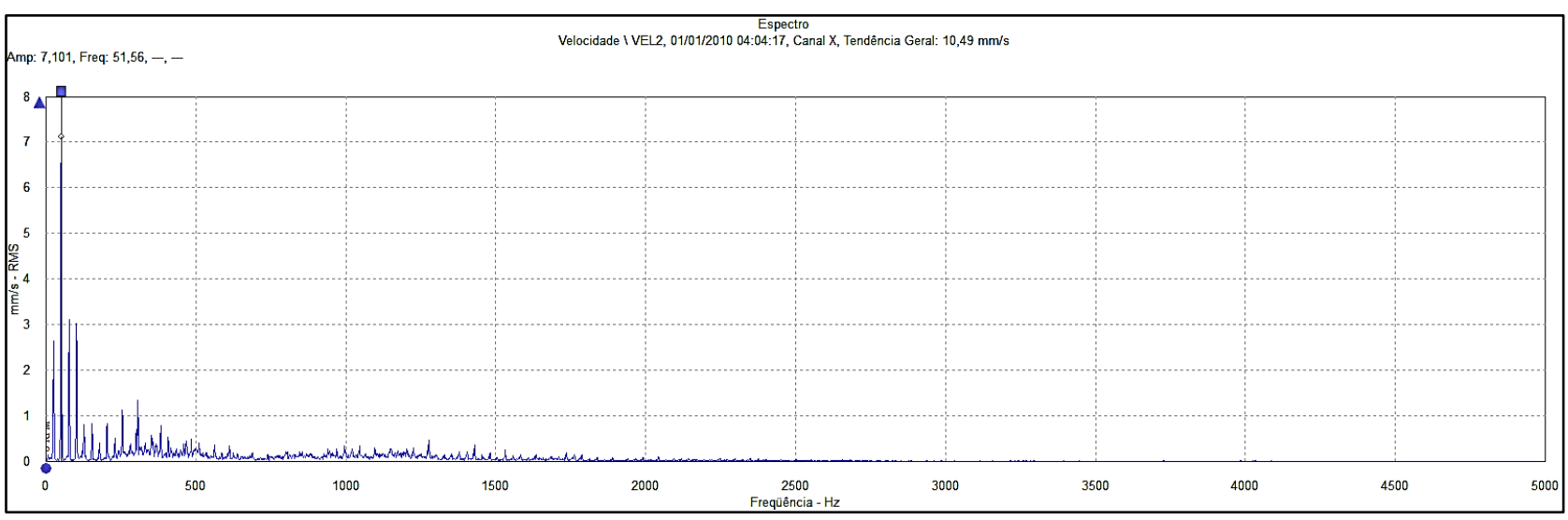

Figura 7. Velocidade horizontal com desbalanceamento a $180^{\circ}$. Fonte: Autores.

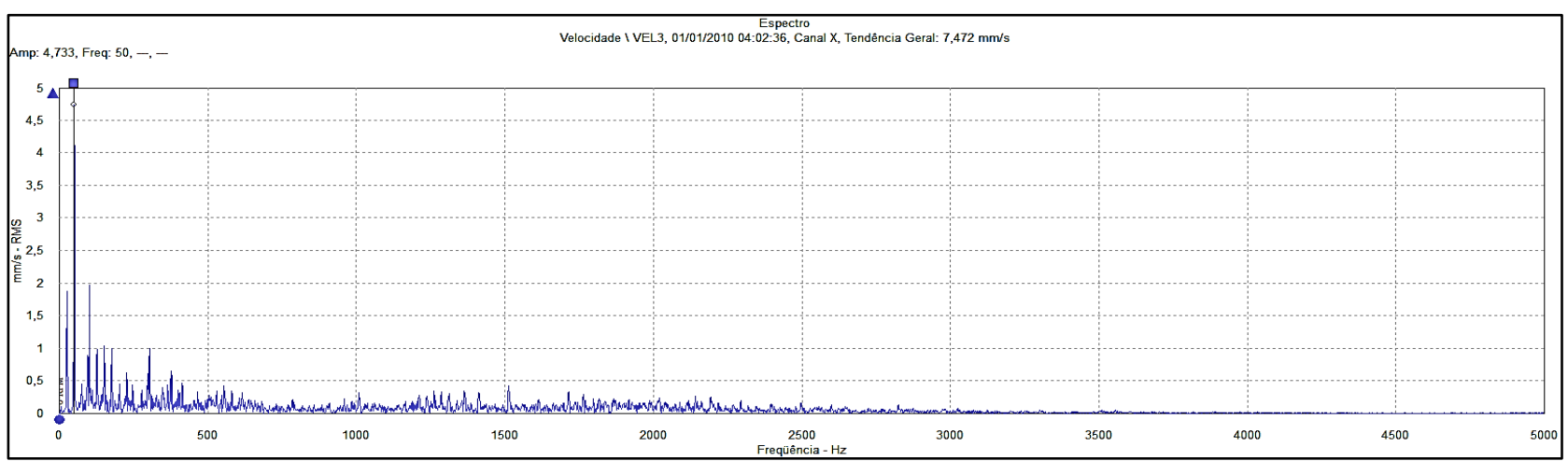

Figura 8. Velocidade axial no modo ideal. Fonte: Autores. 


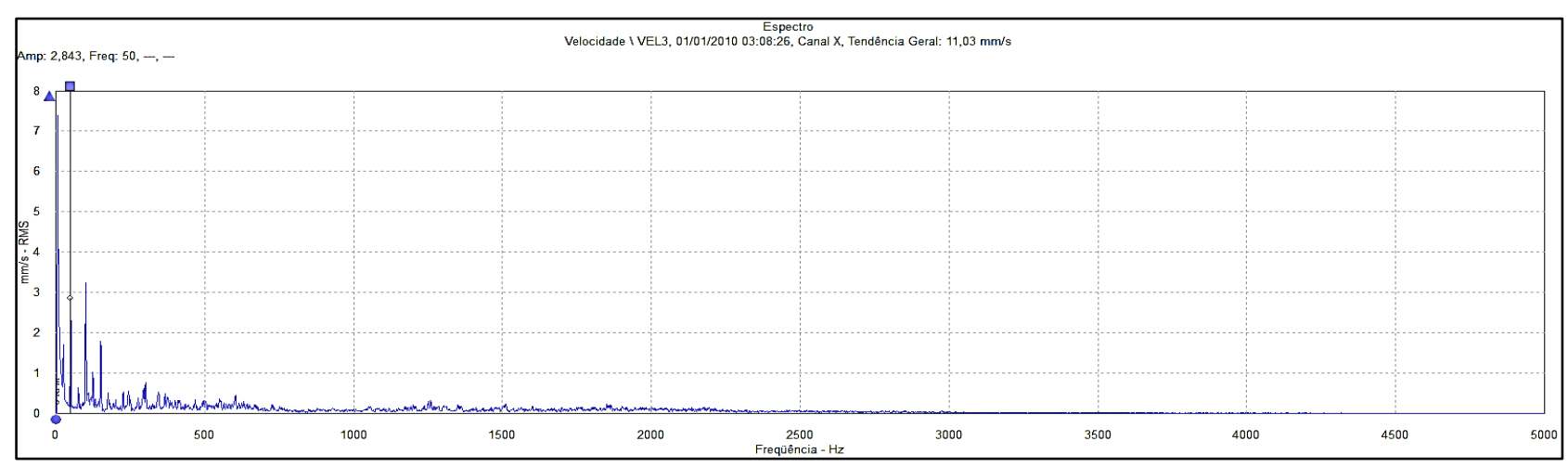

Figura 9. Velocidade axial com desbalanceamento a $270^{\circ}$. Fonte: Autores.

Para facilitar a comparação dos autovalores de energia nas diferentes condições operacionais, foi gerado um gráfico com os respectivos valores de energia para cada nó, com base nas velocidades horizontal e axial.

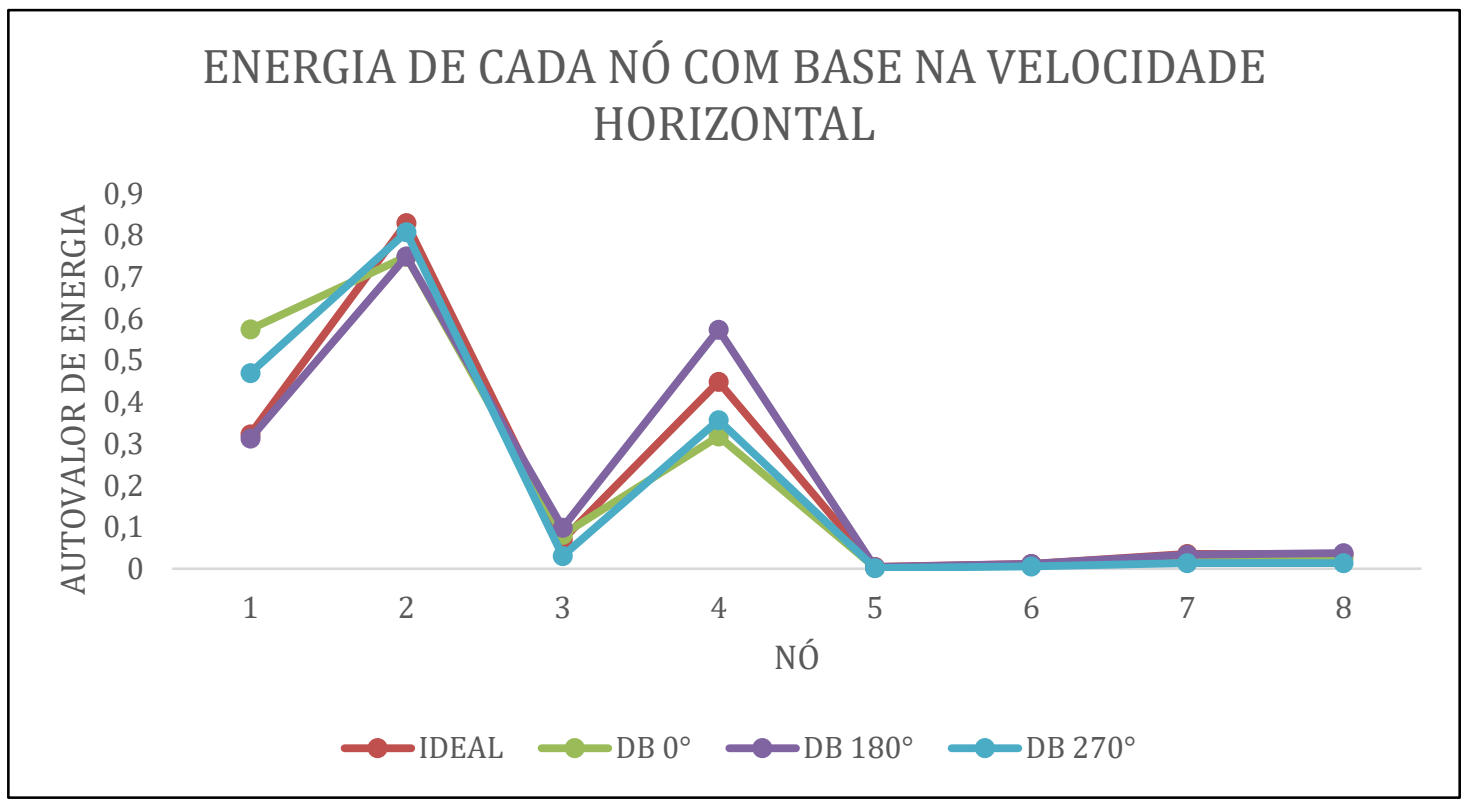

Figura 10. Energia de cada nó com base na velocidade horizontal. Fonte: Autores. 

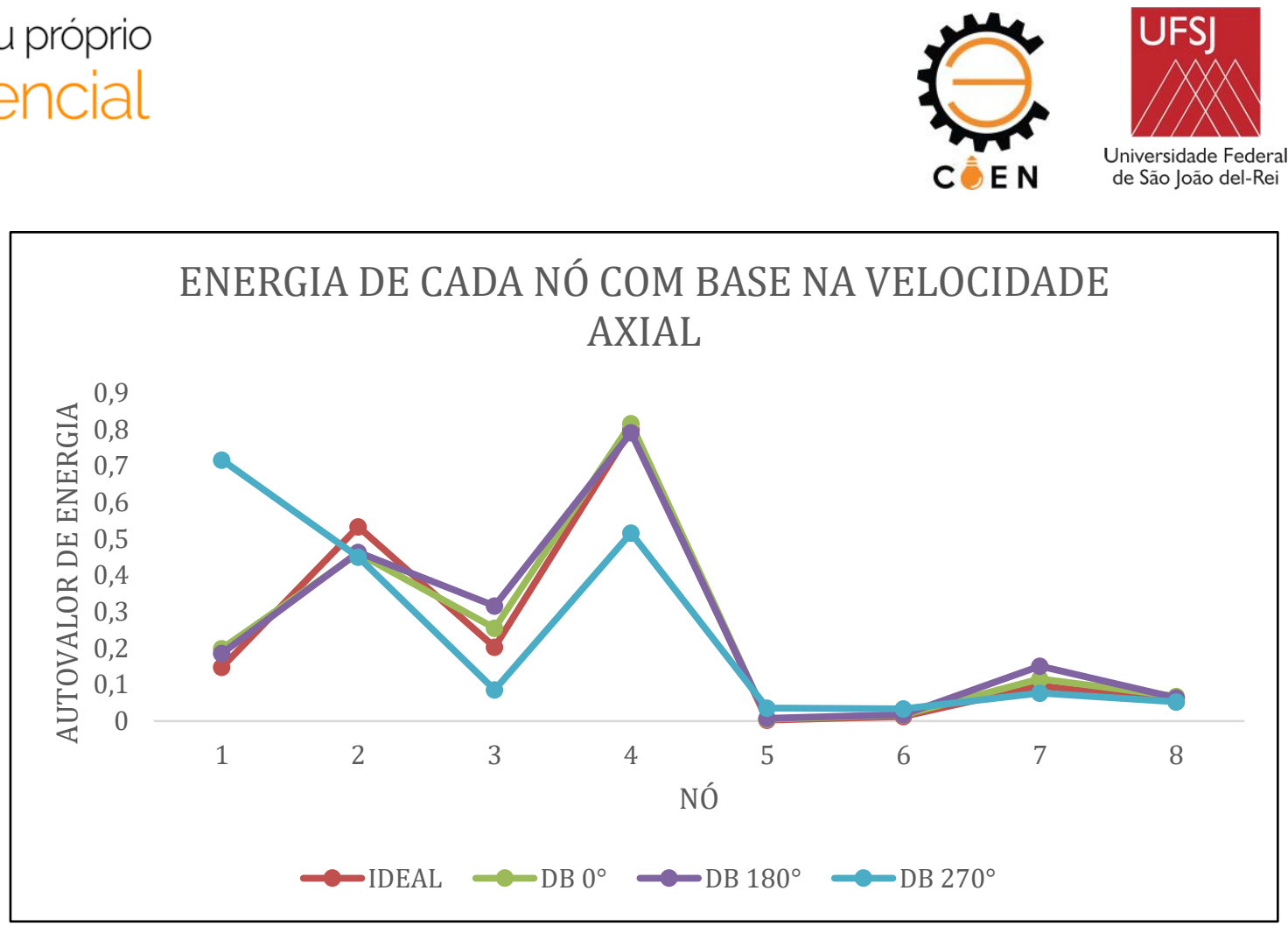

Figura 11. Energia de cada nó com base na velocidade axial. Fonte: Autores.

O primeiro nó, que compreende a faixa de 0 a $800 \mathrm{~Hz}$, é o que está relacionado com as frequências características do motor, tais como engrenamento, rotação e ciclo mecânico. $\mathrm{O}$ segundo nó, está relacionado com as frequências moduladas por impactos fluidodinâmicos. O terceiro nó traz informações provenientes da faixa de frequência que vai de $2400 \mathrm{~Hz}$ a $3200 \mathrm{~Hz}$. A faixa de frequência que vai de $1600 \mathrm{~Hz}$ a $2400 \mathrm{~Hz}$ é representada pelo nó 4, onde ainda há presença de frequência modulada e possivelmente a sua propagação, que se dá de forma aleatória derivando do processo de ignição (Assis, 2018).

A análise dos espectros e dos gráficos de autovalores de energia permite visualizar que na posição horizontal, com desbalanceamento a $180^{\circ}$, o autovalor cresce na banda de frequência correspondente ao nó 4. Entretanto, para a primeira banda de frequências, a vibração se assemelha ao modo ideal de vibração, o que é evidenciado também pelo gráfico de autovalores. Analisando a energia com base na velocidade axial, nota-se que o valor proveniente do desbalanceamento a $270^{\circ}$ é o mais alto no primeiro nó, entretanto ele é o menor para os nós 2, 3 e 4 . Ao observar os espectros de vibração no modo ideal e o que é decorrente do desbalanceamento a $270^{\circ}$, é evidente uma diminuição das amplitudes vibracionais acima de $800 \mathrm{~Hz}$, o que coincide com o visto pelo método da Transformada de Wavelet. 


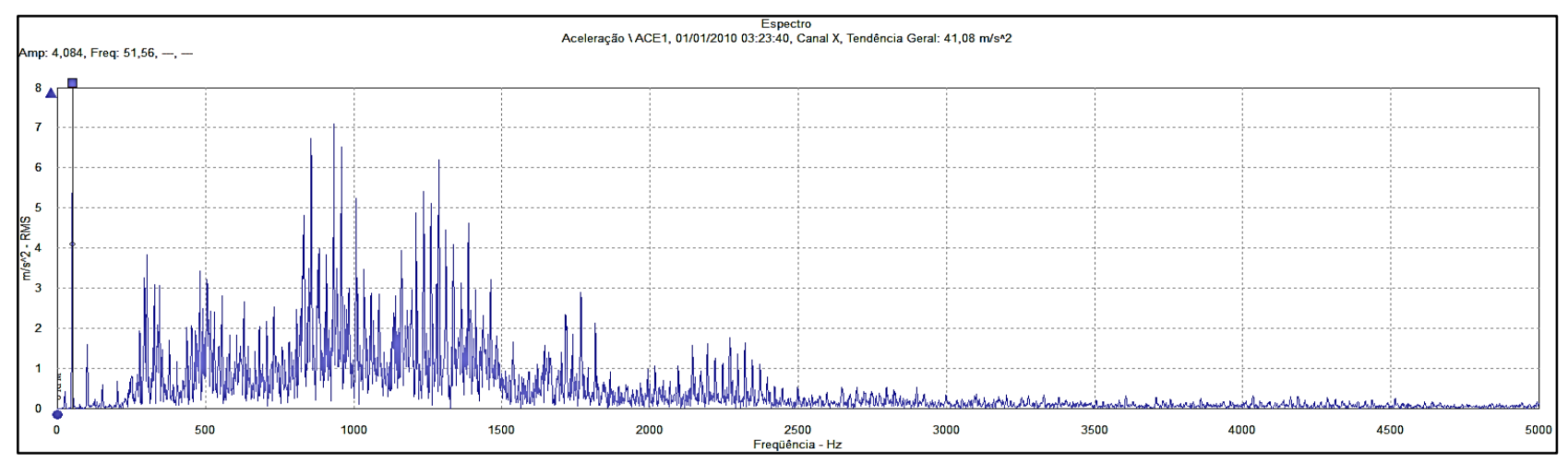

Figura 12. Aceleração vertical no modo ideal. Fonte: Autores.

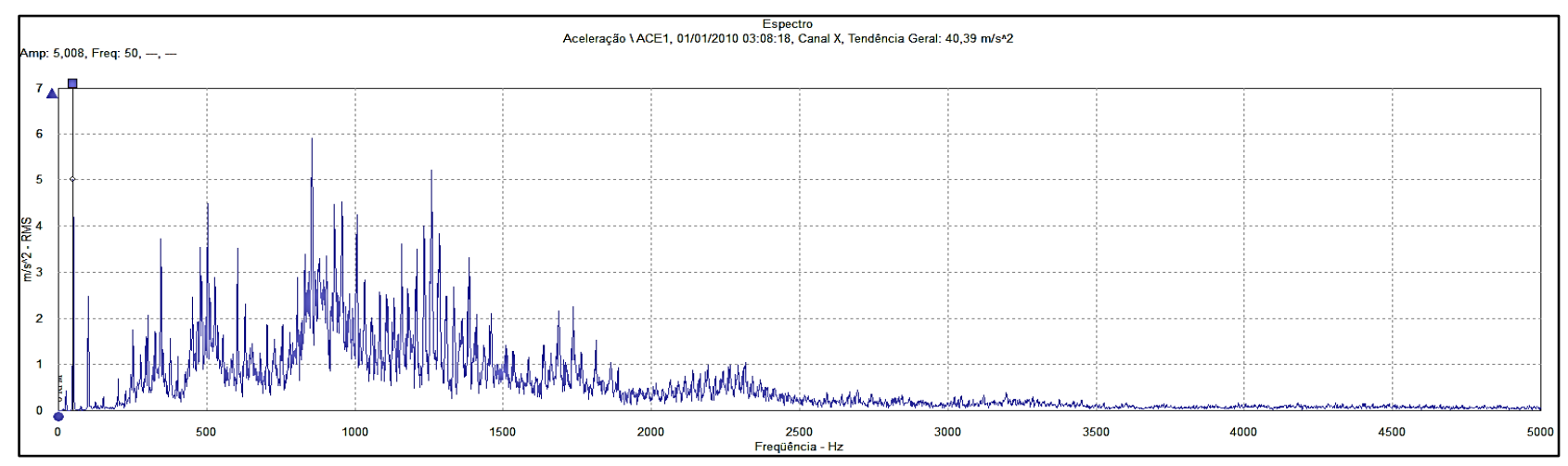

Figura 13. Aceleração vertical com desbalanceamento a $270^{\circ}$. Fonte: Autores

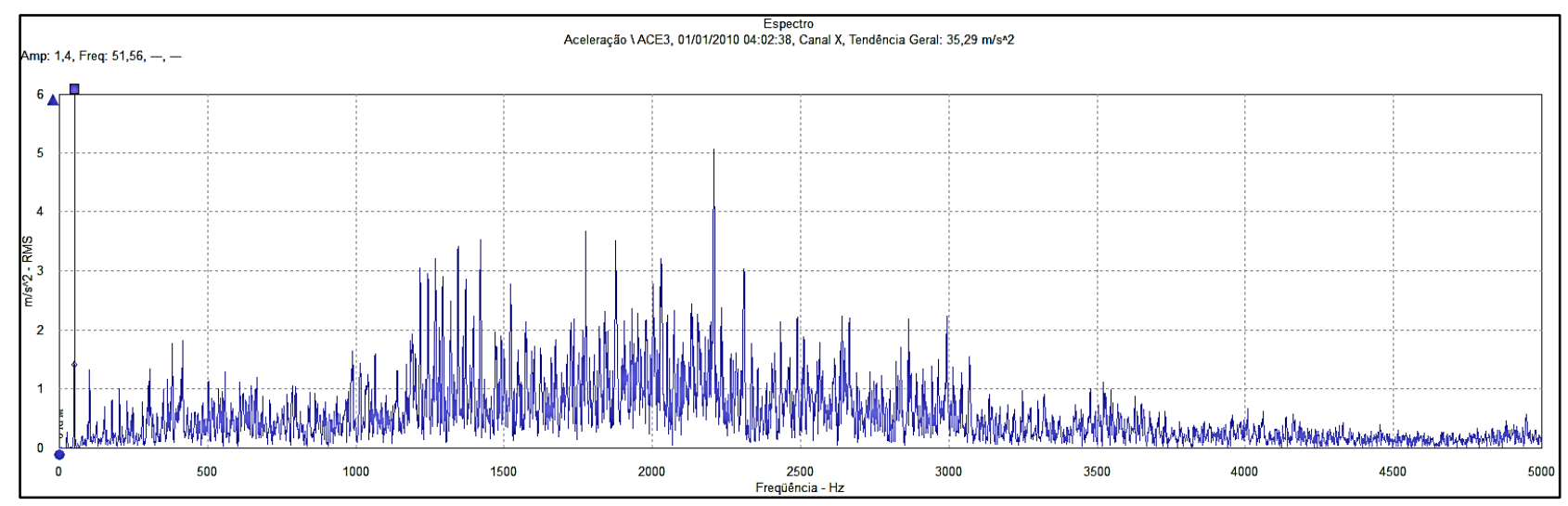

Figura 14. Aceleração axial no modo ideal. Fonte: Autores. 


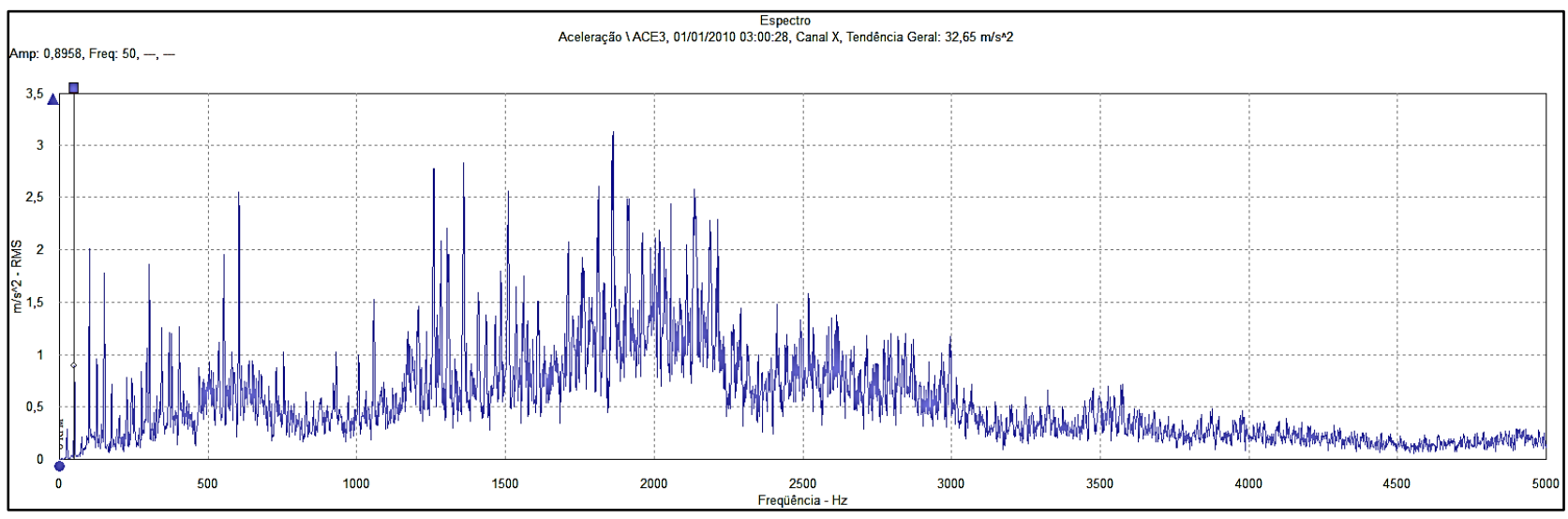

Figura 15. Aceleração axial com desbalanceamento a $270^{\circ}$. Fonte: Autores.

Para a análise e caracterização por meio da aceleração, foram gerados os gráficos de autovalores com base na aceleração das posições vertical e axial, que foram as posições que apresentaram discrepâncias relevantes a este estudo.

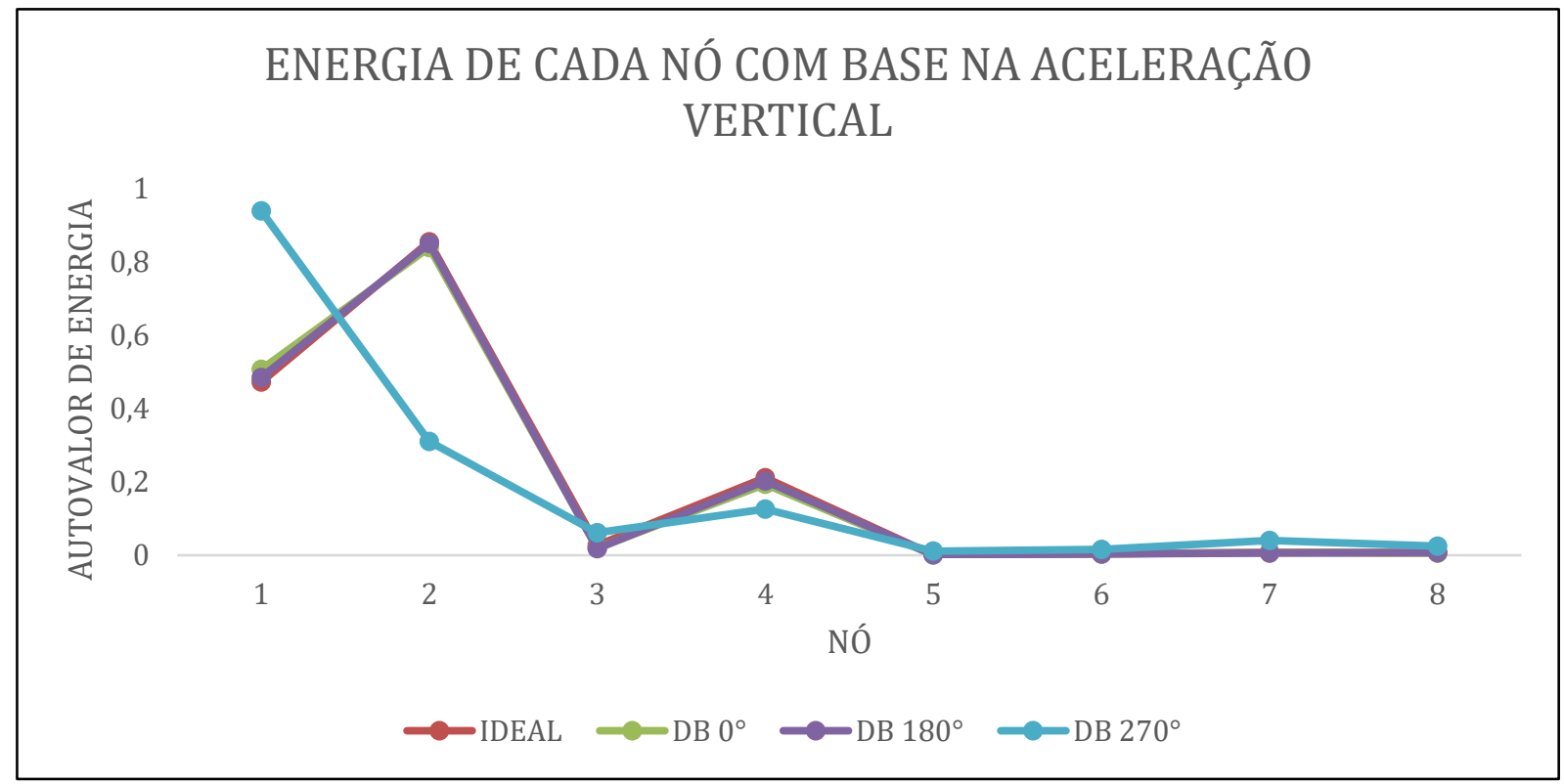

Figura 16. Energia de cada nó com base na aceleração vertical. Fonte: Autores. 

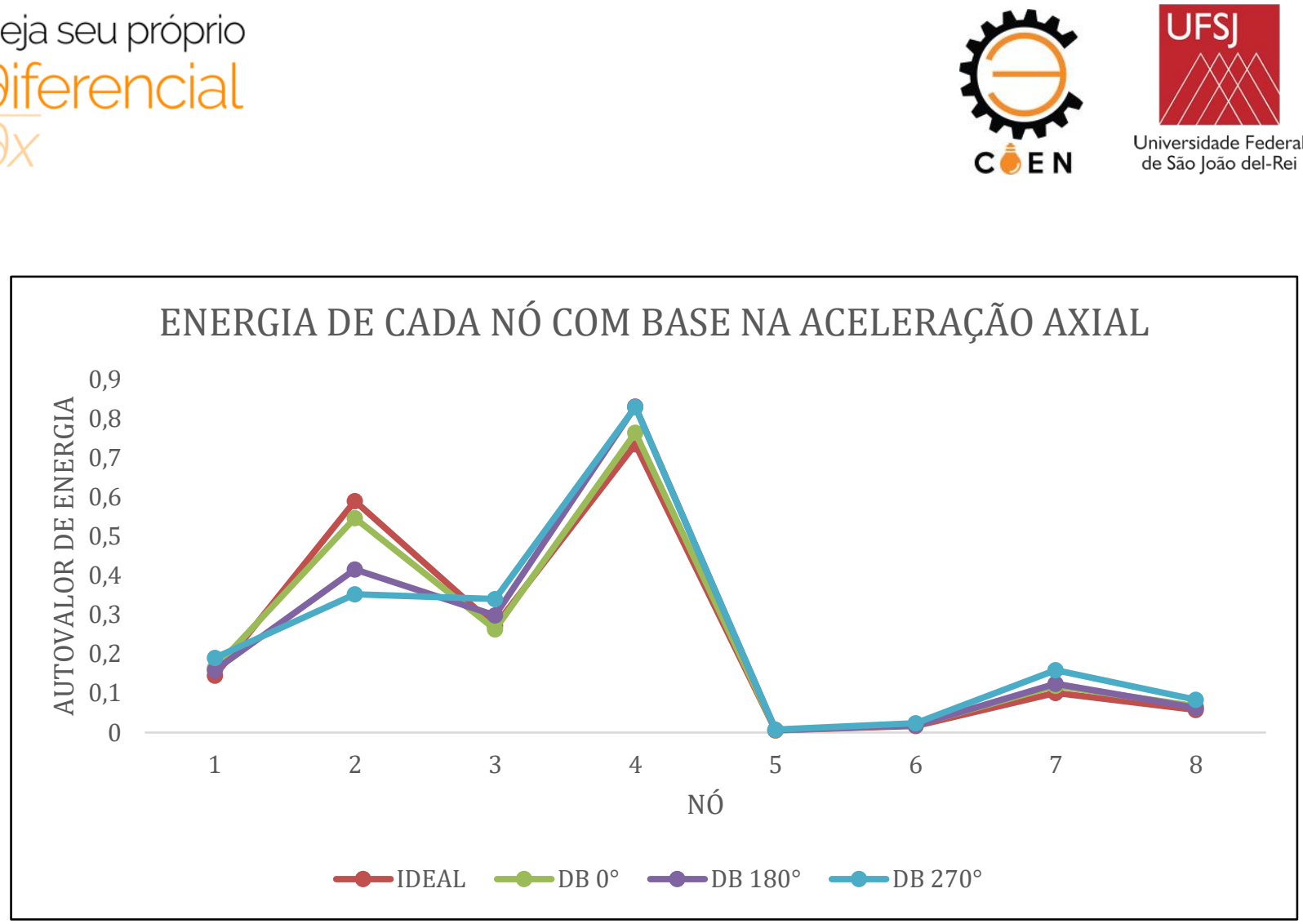

Figura 17. Energia de cada nó com base na aceleração axial. Fonte: Autores.

A análise dos gráficos de autovalores de energia permite visualizar que na posição vertical, com desbalanceamento a $270^{\circ}$, o autovalor tem uma queda considerável do primeiro para o segundo e terceiro nós, tendo seu valor elevado no nó 4, mas ainda sim permanece abaixo dos demais.

Analisando a energia com base na aceleração axial, nota-se que o valor proveniente do desbalanceamento a $270^{\circ}$ é o mais alto no primeiro nó, entretanto ele o menor para o nó 2 , voltando a ser o de valor mais alto em 3 e 4 .

Observando os espectros vibracionais é possível perceber a elevação do carpete para os dados com desbalanceamento a $270^{\circ}$. Em motores elétricos, tal elevação pode indicar deficiência de lubrificação. Para motores a combustão interna ainda não se tem dados consolidados que indiquem o modo de falha representado por este tipo de comportamento. A amplitude dos picos é reduzida, sobretudo para valores acima de $1600 \mathrm{~Hz}$, onde os picos são provenientes de propagação de frequências moduladas derivadas do processo de ignição. Este fato pode estar associado à compensação do desbalanceamento no processo de compressão e/ou exaustão dos gases na câmara de combustão.

Ainda não é possível afirmar categoricamente que o comportamento vibracional para desbalanceamento de motores a combustão interna sempre se dará dessa forma, seria necessário estender o teste para outros modelos. Entretanto, devido à repetitividade nos testes e valores obtidos durante este estudo é possível afirmar que para o modelo Robin EH17-2D, a utilização de Transformada de Wavelet permitiu caracterizar de forma eficaz a vibração do motor, em condição ideal de operação e para desbalanceamento nas posições $0^{\circ}, 180^{\circ}$ e $270^{\circ}$, tornando possível o acompanhamento preditivo deste tipo de falha. 

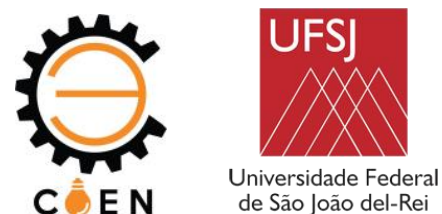

\section{DIREITOS AUTORAIS.}

Os autores são os únicos responsáveis pelo conteúdo das informações contidas neste artigo.

\section{REFERÊNCIAS}

ASSIS, E.K.; LACERDA, A.L.M.; CARVALHO. A.V.; SILVA, J.A.; BRITO, J.N. Análise Do Comportamento Vibracional De Um Motor De Ignição Por Centelha Pelo Método Da Transformada De Wavelet. Anais do XVIII Congresso Nacional de Engenharia Mecânica e Industrial. Joinville-SC, Brasil, 2018.

BRITO, J. N. Desenvolvimento de um sistema inteligente híbrido para diagnóstico de falhas em motores de indução trifásicos. Campinas: Universidade Estadual de Campinas, 214p. Tese (Doutorado), 2002.

GENG, Z.; CHEN, J.; HULL, J.B. Analysis of engine vibration and design of an applicable diagnosing approach. International Journal of Mechanical Sciences 2003; 45: 1391-1410.

GOLWINE, G., BOTTON, G., RIVIN, B., AND SHER, E., Assesment of the Quality of Combustion in Compression Ignition Engines through Vibration Signature Analysis, Fisita World Automotive Congress, Barcelona, 2004.

LACERDA, A.L.M. Estudo do Nível de Vibração do Motor Ciclo Diesel Monocilíndrico na Combustão do Diesel e do Biodiesel Através da Análise de Wavelet. Trabalho de Conclusão de Curso - Departamento de Engenharia Mecânica da Universidade Federal de São João Del Rei, 2014. 93 p.

LACERDA, A.L.M.; LAMIM FILHO, P.C.M.; BRITO, J.N.; PEDERIVA, R. Detection of Faults in Three Phase Induction Motors Using Wavelet Packet Analysis. Proceedings of the 21st Brazilian Congress of Mechanical Engineering, Natal-RN, Brasil, 2011.

PORFÍRIO,G.D. Utilização das Transformadas Wavelets na Detecção de Características em um Sinal de ECG, Projeto de Pesquisa, Universidade Federal de Itajubá, Itajubá, 2011.

REZENDE, J. C. F. Manutenção Preditiva em Turbomáquinas utilizando Sistemas Especialistas na Análise de Vibração. Tese de Mestrado. COPPE/UFRJ, Universidade Federal do Rio de Janeiro, Rio de Janeiro. 1991.

SILVA, V.A.D. Detecção de falhas em motores elétricos através das máquinas de vetores de suporte. Dissertação de Mestrado em Engenharia Mecânica- Universidade Estadual de Campinas, Faculdade de Engenharia Mecânica. - Campinas, SP: [s.n.], 2012.

\section{STUDY OF VIBRATIONAL ENGINE BEHAVIOR INTERNAL COMBUSTION SUBMITTED TO UNBALANCE IN DIFFERENT POSITIONS BY WAVELET TRANSFORMING}

Elen Kássia de Assis ${ }^{(1)}$ (elen.kassia@engenharia.ufjf.br), Marcel Henrique Lopes ${ }^{(1)}$ (marcel.henrike@hotmail.com), Jorge Nei Brito ${ }^{(2)}$ (brito@ufsj.edu.br), José Antônio da Silva ${ }^{(3)}$ (jant@ufsj.edu.br)

\footnotetext{
(1) Univ. Federal de São João Del Rei (UFSJ) - Grupo de Estudos e Pesquisas do Laboratório de Sistemas Dinâmicos (Gep_LASID)

(2) Universidade Federal de São João Del Rei (UFSJ) - Departamento de Engenharia Mecânica (DEMEC)

(3) Universidade Federal de São João Del Rei (UFSJ) - Departamento de Ciências Térmicas e dos Fluidos (DCTEF)
}

ABSTRACT: Vibration analysis is one of the most commonly used predictive maintenance methods for fault identification. It is widely used for electric motors, however its application to internal combustion engines is more complex, due to the difficulty in developing a standard. This study aims to characterize the vibrational pattern of the Subaru Robin EH17-2D engine based on different unbalance positions. The vibrational data 
were collected with the IMX-S 16 equipment from SKF with the help of piezoelectric accelerometers. The vibrational spectra were generated from @ ptitude Analyst software, also from SKF. MatLab software was used to implement a signal processing algorithm through the Wavelet Transform, which allowed the characterization of the vibrational energy of each frequency band. For the graphical exhibition in the work, the positions in which the energy values and the changes in the vibrational pattern were shown to be more relevant were selected. Knowing the operant mode of the motor in conditions prone to failure, it is possible, through predictive monitoring, to identify, when it has anomalous frequencies, that there is an imminent fault.

KEYWORDS: Predictive maintenance. Vibration. Internal combustion engine. Imbalance. Wavelet Packet Transform. 